\title{
Should refinement 'mess with success'?
}

Dr. Ted Clark studied dietary cholesterol metabolism and drugs for controlling serum cholesterol levels. The animal model he used was the owl monkey (Aotus nancymaae) and a key part of most of his protocols included a cholecystectomy, the surgical removal of the animal's gallbladder. A new laboratory animal veterinarian at Great Eastern University was prereviewing Clark's most recent protocol. At first, she wondered if the cholecystectomy was really needed but Clark described the rationale in sufficient detail to convince the veterinarian that the surgery was required. However, the veterinarian questioned why Clark continued to use a relatively large laparotomy incision to remove the gallbladder, while research and clinical experience suggested that one or two small incisions, just large enough for a laparoscope, could be used to achieve a cholecystectomy with less postoperative pain to the animal. Clark responded that he had nearly 20 years of experience with the open abdomen technique but none with laparoscopy. He never lost an animal, he always provided the post-operative analgesia and general care that was approved by the IACUC, and he had a solid record of publications that had never questioned his methodology. He did not understand why now, out of the blue, his methodology was being questioned, and he was being asked to spend a significant amount of research funds to purchase equipment and be trained in a technique that would lead to research results that were no better than those he was already getting.

The IACUC office explained to Clark that the veterinarian was suggesting, not demanding, that a potentially less painful procedure be considered. This did not mollify Clark who saw the suggestion as an intrusion on his academic freedom and as second guessing of both a Principal Investigator and the decision of an NIH study section to fund his research.

What do you think? Should the veterinarian or the IACUC require a better explanation from Clark, or has his success over the years been sufficient proof that his methodology is appropriate for his research? Are there any other avenues for the IACUC to consider?

\section{RESPONSE}

\section{Refinement, a part of a three-legged stool}

\author{
Dinesh K. Hirenallur-S., DVM, MVSc, PhD \& \\ Jennifer McElroy, RVT, LATG, CPIA
}

Since the origin of Russell and Burch's concept of the 3 Rs, replacement, reduction and refinement ${ }^{1}$, investigators and institutions have been following these principles to reduce use and to minimize the distress and discomfort of laboratory animals ${ }^{2}$. In fact, current guidelines such as the Guide for the Care and Use of Laboratory Animals ${ }^{3}$, the PHS policy ${ }^{4}$ and legislative documents including the Animal Welfare $\mathrm{Act}^{5}$ in the United States, and Directive 2010/63/EU ${ }^{6}$ in Europe encourage scientists and institutions to recognize and apply the 3 Rs not only during studies but also during the planning stages of studies, when the animal use protocol is being developed. Of the $3 \mathrm{Rs}$, refinement is the one that has the greatest effect of minimizing pain and distress in laboratory animals used for studies.

According to the Animal Welfare Act, the IACUC should determine that the principal investigator has considered alternatives to procedures that may cause more than momentary or slight pain or distress to the animal ( $\$ 2.31(\mathrm{~d})$; ref. 5). Therefore, in our opinion, the new veterinarian at Great Eastern University was justified both in questioning Clark's invasive approach for cholecystectomy and in suggesting the novel, alternative laparoscopy approach, which would be less invasive. The concept of refinement is a dynamic process that constantly evolves as new technologies and inventions become available. On the other hand, it is reasonable to expect resistance from an investigator when suggesting refined methods if his methods are already working well. The burden often lies with the IACUC and its institution to convince Principal Investigators and implement refinement approaches to minimize distress and discomfort in laboratory animal procedures.

In this particular scenario, the IACUC committee should recommend that Clark consult with the institution's veterinarian to weigh the pros and cons of the suggested laparoscopic cholecystectomy approach. Based on available literature, the veterinarian should provide to Clark objective evidence of the advantages of the laparoscopic approach, such as less postoperative pain, faster recovery and less experimental variability. The veterinarian should also provide a scientific explanation as to why the newer approach would not affect the scientific goal of Clark's study.

Similarly, the veterinarian and IACUC should provide an opportunity for Clark to express his concerns, and Clark should give a scientific rationale to support his views. Further, we don't think that Clark's explanation regarding the success of his past surgical approach provides sufficient justification to disregard the veterinarian's suggestion to use a new, less invasive approach that will minimize animal discomfort and pain. Also, the monetary cost does not constitute a scientific justification for not adapting a suggested refinement technique. If Clark is still concerned about his scientific outcome, the IACUC should 